\title{
The protective role of protocol biopsy against chronic kidney disease progression in kidney transplantation
}

\author{
Okjoo Lee ${ }^{1}$, Kyo Won Lee ${ }^{1}$, Jae Berm Park', Jung Eun Lee ${ }^{2}$, Na Young Hwang ${ }^{3}$, Kyunga Kim ${ }^{3}$ \\ ${ }^{1}$ Division of Transplantation, Department of Surgery, Samsung Medical Center, Seoul, Korea \\ ${ }^{2}$ Division of Nephrology, Department of Internal Medicine, Samsung Medical Center, Seoul, Korea \\ ${ }^{3}$ Department of Biostatistics, Samsung Medical Center, Seoul, Korea
}

Background: Subclinical rejection is associated with chronic allograft nephropathy, which is the most common cause of allograft failure in kidney transplantation (KT). Therefore, early detection and treatment of subclinical rejection through protocol biopsy can reduces the incidence of chronic allograft nephropathy and the improvement of graft survival. This study aims to evaluate the effective early detection role of routine protocol biopsy.

Methods: We retrospectively analyzed 1,361 KT recipients in Samsung Medical Center between July 2007 and August 2017. Of these, pediatric cases, re-transplantations and multi-organ transplantation, cyclosporine and azathioprine users, patients who were not underwent protocol biopsy, and diagnosed rejection but not treated patients were excluded. Finally, a total of 854 adult patients who were underwent protocol were analyzed.

Results: Patients were divided into three groups: no protocol biopsy $(n=350)$, single protocol biopsy group $(n=207)$, and double protocol biopsy group $(n=297)$. Protocol biopsy group was significant higher donor and recipient age, presence of recipient diabetes mellitus and donor hypertension, presence of donor specific antigen, and ABO incompatible KT. Protocol biopsy group showed significant difference from no protocol biopsy group in the tendency of graft function (estimated glomerular filtration rate), and protective result was shown in chronic kidney disease (CKD) progression. Especially, double protocol biopsy group showed the better protective result not only in CKD progression but also in new onset CKD. But Kaplan-Meier curve showed that protocol biopsy has not significantly benefit in graft survival and patient overall survival.

Conclusions: Protocol biopsy can play a protective role in CKD progression in KT recipients. 